\title{
AN APPLICATION OF THE ADDITION THEOREM FOR DETERMINANTS
}

\author{
by HENRY JACK \\ (Received 30th June 1962)
}

THE integral evaluated in this note was suggested by the famous one connected with the Poincaré polynomials of the classical groups (see (1)).

Let $X$ be an $n \times n$ matrix whose elements depend on $k$ parameters. Denote by $\mathscr{X}$ a manifold in Euclidean space of dimension $n^{2}$, with the property that if $X \in \mathscr{X}$, then so does $X I_{-i}$ for $1 \leqq i \leqq n$, where $I_{-i}$ is the unit matrix $I$ altered by a minus sign in the $(i, i)$ th place. Suppose further that there exists on $\mathscr{X}$ a measure which is invariant under the transformation $X \rightarrow X I_{-i}$. Such manifolds and measures exist. For example (see (2), $\S 5$ ), the set of all proper and improper $n \times n$ orthogonal matrices $H$ is such a manifold, the $H$ depending on $\frac{1}{2} n(n-1)$ parameters because of the orthogonality and normality of the columns of $H$. Since the set of all $H$ is a compact topological group, an invariant measure exists.

Theorem. If $d X$ is an invariant measure on $\mathscr{X}$, such that $V=\int_{\mathscr{X}} d X$ exists and is finite, and if $A, B, C$ are constant $n \times n$ matrices, and $|M|$ is the determinant of $M$, then

$$
\int_{x}|A+B X C| d X=V|A| \text {. }
$$

Proof. Suppose $|C| \neq 0$, and let $D=A C^{-1}$, then

$$
\int|A+B X C| d X=|C| \int|D+B X| d X .
$$

Since the measure $d X$ is invariant under the transformation $X \rightarrow X I_{-i}$,

$$
\begin{aligned}
J=\int|D+B X| d X=\int\left|D+B X I_{-i}\right| & d X \\
& =\frac{1}{2} \int\left\{|D+B X|+\left|D+B X I_{-i}\right|\right\} d X .
\end{aligned}
$$

Now $|D+B X|$ and $\left|D+B X I_{-1}\right|$ differ only in their first columns, so their sum is a determinant $\left|2 d_{1},(D+B X)_{n-1}\right|$, whose first column is twice the first column, $d_{1}$, of $|D|$ and whose remaining columns $(D+B X)_{n-1}$, are the last $n-1$ columns of $|D+B X|$. So

$$
J=\int\left|d_{1}, \quad(D+B X)_{n-1}\right| d X .
$$


Now carry out the transformation $X \rightarrow X I_{-2}$ and let $d_{2}$ be the second column of $|D|$ and

Continuing,

$$
J=\int\left|d_{1}, d_{2}, \quad(D+B X)_{n-2}\right| d X .
$$

$$
J=\int\left|d_{1}, \quad d_{2}, \quad \ldots, \quad d_{n}\right| d X=V|D| .
$$

This proves the theorem when $|C| \neq 0$. But when $|C| \neq 0,(1)$ is an identity between two polynomials in the elements of $C$, and so by continuity, it still holds when $|C|=0$.

Corollary. Let $E_{r}(X)$ be the elementary symmetric functions of the latent roots of $X$, then

$$
\int_{\mathscr{X}} E_{r}(X) d X=0 .
$$

Proof. Let $A=z I, B=C=I$. Then since

$$
\begin{aligned}
|z I| & =z^{n} \text { and }|z I+X|=z^{n}+\sum_{r=1}^{n} z^{n-r} E_{r}(X), \\
\sum_{r=1}^{n} z^{n-r} \int E_{r}(X) d X & =0 \text { for any number } z .
\end{aligned}
$$

\section{REFERENCES}

(1) D. E. Litrlewood, On the Poincaré polynomials of the classical groups, Journ. London Math. Soc. 28 (1953), 494-500.

(2) H. JACK and A. M. MACBEATH, The volume of a certain set of matrices, Proc. Camb. Phil. Soc. 55 (1959), 213-223.

Queen's College

DUNDEE 